\title{
Desarrollo y escalamiento productivo de un nuevo surfactante pulmonar
}

\section{Development and productive scaling of a new pulmonary surfactant}

Vicente Rangel,

Evelyn Arias,

Raimundo Rojas,

Adriana Luque,

Pedro Bortot,

Germán Da Ruos,

Elisamelis Martínez,

Julissa Antequera,

Iván Sojo,

Tomás Bonillo,

Laura Hernández,

Dolores Clemente,

José Rangel,

Rodolfo Vargas.

Unidad de Estructura Molecular, Fundación Instituto de Estudios Avanzados (IDEA), Venezuela

Graciela Lucia Uzcanga

Unidad de Estructura Molecular, Fundación Instituto de Estudios Avanzados (IDEA), Venezuela

Universidad Internacional SEK, Ecuador

Autor por Correspondencia: graciela.uzcanga@uisek.edu.ec

Fecha de recepción: 14 de Abril de 2017 - Fecha de aceptación: 30 de Junio de 2017

Resumen: Este trabajo es una guía referencial para el desarrollo de nuevos productos biofarmacéuticos y se basa en la experiencia obtenida del desarrollo de un nuevo Surfactante Pulmonar. Mediante las estadísticas de nacimientos prematuros, se estimó que la demanda del producto en los países del ALBA es de 45.000 viales de $120 \mathrm{mg}$ /vial por año para el año 2007. Para la extracción y purificación del nuevo surfactante, a partir pulmones de porcinos, se desarrolló un protocolo novedoso que es aplicable a nivel industrial. El medicamento consiste en una sustancia biológica liofilizada, clasificada como polvo estéril para reconstituir, formulado para resuspenderse en $3 \mathrm{ml}$. de agua para inyectables, para su aplicación por vía endotraqueal. Los proveedores de la materia prima son mataderos industriales, certificados por la autoridad sanitaria (MPPS), y por la autoridad ambiental (MINAMB). Para garantizar que el sistema productivo mantenga los atributos de calidad del medicamento, desde la producción, pasando por su distribución en el mercado, hasta la utilización del surfactante en neonatos, se diseñó una infraestructura apropiada y calificada para la producción masiva. El proceso para la industrialización del medicamento se basa en la normativa sanitaria vigente, que exige el control de todas sus fases: concepción, diseño, construcción, equipamiento, adecuaciones, puesta en marcha y funcionamiento de la planta. Este control implica el establecimiento de un Sistema de Aseguramiento de la Calidad, con alcance a todos los ámbitos de la empresa fabril y su cadena de distribución, que garantice la ejecución continua y consistente de todos los procesos.

Palabras claves: biofarmacéuticos; transferencia de tecnología; aseguramiento de la calidad; buenas prácticas de manufactura (BPM) 


\begin{abstract}
On the basis of premature births statistics, the pulmonary surfactant demand is estimated to be 45,000 vials of $120 \mathrm{mg}$ each, per year, in the ALBA countries. Here, we developed a new pulmonary surfactant protocol, which can be applied to an industrial scale. The pulmonary surfactant was produced from pig lungs and is available as a sterile freeze-dried powder that only requires to be reconstituted with $3 \mathrm{ml}$ of sterile water before its use. The product should be administered into the lungs by an endotracheal tube. Raw material suppliers were slaughterhouses certified by the health authority (MPPS) in terms of good manufacturing practices for food, and certified by the environmental authority (MINAMB). In addition, the infrastructure was designed, complying with all the health authority requirements, to ensure that the production system maintained the surfactant quality and properties along the whole process: during the extraction, throughout the distribution market, and when used by the final consumers. Therefore, the industrialization process is also based on health regulations, which require controls at all stages: conception, design, and construction, equipment's, adjustments, and commissioning and plant operation. These controls involve the establishment of a quality assurance system, which must reach all manufacturing company areas and distribution system. The quality assurance system contemplates the qualification of the critical components and systems to assure the continuous and consistent execution of functions.
\end{abstract}

Keys words: biopharmaceutical; technology transfer; quality assurance system; good manufacturing practices

\title{
Introducción
}

La Industria Farmacéutica es uno de los sectores industriales más robustos y de mayor auge a nivel mundial. La Organización Mundial de la Salud (OMS), a través de su Comité de Expertos, emitió en 1992, las Normas de Buenas Prácticas de Manufactura (BPM), en su Informe 32, en respuesta a la necesidad de controlar todo el sector farmacéutico. A través de nuevos informes, la OMS ha ido reglamentando cada uno de los factores involucrados en la producción de medicamentos. La industrialización de los procesos productivos de medicamentos ha ido ganando terreno, y el Aseguramiento de la Calidad se ha afianzado como una nueva filosofía gerencial. De las unidades de investigación y desarrollo (I \& D) de las empresas farmacéuticas o de las inversiones de estas en laboratorios de investigación, ha surgido la gran mayoría de los nuevos desarrollos farmacéuticos. En cuanto a los nuevos desarrollos llevados por particulares, sus oportunidades para industrializarse son muy pocas, por lo que muchos de ellos son adquiridos por grandes empresas del sector. En lo que respecta al sector público, ha sido tendencia de algunos gobiernos, la adquisición de las patentes de algunos medicamentos para su manufactura y colocación en el mercado, como medicamentos gratuitos o a precios accesibles.

Los avances de la bio-ingeniería, los descubrimientos genéticos y de estructura del ADN, han permitido el desarrollo de nuevas líneas de investigación en el mundo, siendo unas de ellas las líneas de Investigación Biofarmacéutica. Las nuevas soluciones a los problemas de la salud mundial, se encuentran en los avances de las investigaciones de las ciencias de la biología y la medicina; estos avances han sido denominados desarrollos en Biotecnología (Medicamentos Biotecnológicos y Biosimilares). Los Medicamentos Biológicos son productos que poseen una o varias sustancias activas, las cuales se derivan de un organismo viviente, es decir, tales como los microorganismos; órganos y tejidos de origen animal o vegetal, y células o fluidos de origen humano o animal; entre otros. 
Los Biotecnológicos, por su parte, son productos biológicos/tecnológicos que se obtienen a través de la tecnología del ADN recombinante. La complejidad en la fabricación de productos biofarmacéuticos es debido a su naturaleza lábil (inestable), inherente a su origen en organismos vivos, son sensibles a cambios en el proceso de fabricación, lo cual hace mucho más exigente los protocolos de control de calidad del ente regulador para garantizar la unicidad en cada producto. Existen riesgos serios y diversos con este tipo de productos debido a su inmunogenicidad, y a la posibilidad de transferencia de patógenos. Es indispensable que la seguridad y eficacia de los biofármacos esté garantizada con procedimientos más estrictos y profundos que los aplicados a la certificación de un producto genérico químico.

La obtención de materia prima debe cumplir con varios requisitos establecidos, en la Ley de Medicamentos para las plantas de producción de hemoderivados y afines, ajustándose estrictamente a las Normas de Buenas Prácticas de Manufactura de La Organización Mundial de La Salud, Normas de Buenas Prácticas de Manufactura del Ministerio de Salud y Desarrollo Social, Normas de Buenas Prácticas de Manufactura de Productos Cosméticos. La European Medicines Agency (EMEA) ha declarado que los medicamentos biosimilares son productos realmente únicos, no genéricos, ni copias idénticas de productos biológicos originales y que por lo tanto es indispensable que la información al médico y al paciente sea clara y completa.

El estado venezolano ha incursionado discretamente en este sector (el farmacéutico), con productos sumamente necesarios y de gran impacto en la salud del venezolano. De allí, la importancia que tiene el desarrollo, a nivel gubernamental, de proyectos conducentes a la industrialización de medicamentos, así como en lo referente a Investigación y Desarrollo (I+D), y de la aplicación de estrategias para la colocación de estos medicamentos al alcance del colectivo.

El Poder Legislativo adoptó el Informe 32, por medio de la Gaceta Oficial número 38.009, del 26 de agosto de 2004, de cumplimiento obligatorio por todas las empresas del sector a nivel nacional, que especifica los siguientes elementos esenciales para la administración de la calidad en la industria farmacéutica, los cuales deben ser tomados en cuenta para el desarrollo de un proyecto de industrialización de un medicamento de innovación: Garantía de la calidad, Prácticas adecuadas de fabricación de productos farmacéuticos (PAF), Control de la calidad, Saneamiento e higiene, Validación, Quejas, Retiro de un producto, Producción y análisis por contrato, Autoinspección y auditoría de calidad, Personal. Instalaciones, Equipos, Materiales Documentación. Este instrumento habrá de complementarse con toda la legislación relativa a aspectos clave del funcionamiento de una planta farmacéutica, tales como medio ambiente, mantenimiento de equipos, condiciones de ambiente de trabajo en áreas productivas para protección de materiales en proceso y del personal, manejo de materiales, entre otros. En presente trabajo, resume el procedimiento apegado a las normativas nacionales e internacionales que garantizan la obtención un nuevo biofármaco con la garantía de calidad necesaria para su aplicación en humanos. Cabe destacar que, desde el momento de su concepción del nuevo medicamento es necesario mantener la premisa del desarrollo de un sistema de aseguramiento de la calidad en cada proceso o etapa.

\section{Materiales y métodos}




\section{Información técnica del medicamento.}

Se evaluaron los procedimientos y resultados de las investigaciones realizadas sobre el surfactante pulmonar, métodos de obtención y de análisis de composición y ensayos de evaluación de la eficacia in vivo e in vitro. Se identificaron: el origen, la composición y uso de los surfactantes naturales y la información acerca de la Terapia de Reemplazo de Surfactante (TRS) en estudios clínicos para determinar eficacia de la TRS en el síndrome de distrés respiratorio (SDR).

\section{Estimación de la demanda y evaluación del arte previo.}

El desarrollo del proyecto contempló el calculó de la demanda del medicamento para establecer las metas de producción que se deben alcanzar. Se realizó una estimación de la incidencia del Síndrome de Distrés Respiratorio neonatal en América Latina, con excepción de los países que producen el surfactante (Brasil, Argentina y Cuba), basándose en los registros de nacidos prematuros de menos de 1500 gramos. Se calculó la demanda de surfactante pulmonar en la región, incluyendo no solo los casos de mortalidad, sino los casos que sobreviven al SDR mediante el uso de respiradores artificiales o por el uso de surfactante pulmonar.

Para el análisis, se tomó como criterio el hecho de que el SDR afecta aproximadamente al $25 \%$ de los niños prematuros. Esta cifra se fijó teniendo en cuenta valores de incidencia conocidos de varios países de la región y que no todos los recién nacidos de bajo peso (RNBP) desarrollan esta patología; la frecuencia es mayor en la edad gestacional entre las 28 y las 34 semanas. Vale señalar que en algunos países donde el porcentaje de partos atendidos por personal especializado es bajo, la incidencia del SDR podría ser mayor. Se procedió a la búsqueda de las patentes relativas al producto (fórmulas, protocolos de fabricación y tecnologías de obtención). Los resultados de este análisis sirvieron de base para el manejo de la propiedad intelectual concerniente al medicamento.

\section{Investigación, desarrollo y transferencia de tecnología.}

\section{Obtención de la materia prima biológica.}

La metodología para la obtención de la materia prima biológica consistió en la caracterización del insumo y sus proveedores. Se tomó en cuenta que se trata de un material biológico, se verificaron los Sistemas de Aseguramiento de la Calidad (SAC) de las empresas proveedoras, y se establecieron cooperaciones con el fin de adecuar su infraestructura para el manejo seguro de la materia prima. Adicionalmente, se procedió al diseño del procedimiento para el transporte del material.

\section{Procedimiento de obtención del surfactante pulmonar.}

El procedimiento industrial para la obtención de surfactante pulmonar fue desarrollado en nuestro laboratorio. Este procedimiento cumplió con tres premisas: La primera era obtener un producto con la composición descrita en la literatura en cuanto a la identificación y proporción de proteínas y fosfolípidos, y que demostrase actividad in vivo. La segunda, obtenerlo mediante 
un procedimiento novedoso que no estuviese bajo protección intelectual, es decir, que no estuviese patentado, y el tercero que el tiempo de procesamiento de la materia prima y obtención del surfactante fuese viable en términos de operatividad y estabilidad del producto. El procedimiento se definió mediante la evaluación de tecnologías que fuesen aplicables a nivel industrial, que sustituyeran los pasos que se llevaban a cabo en el laboratorio, manteniendo la composición del surfactante. Con esto, se obtiene el diagrama de flujo del proceso productivo.

Los resultados obtenidos durante el desarrollo del medicamento y de su proceso productivo, fueron la base para la definición del producto terminado, que consistió en el establecimiento de las especificaciones del producto terminado, bajo los siguientes parámetros:

a) Composición físico-química del medicamento: la droga, o principio activo, que ejerce la acción terapéutica, y los excipientes.

b) Empaque primario: descripción y dimensiones del material en contacto con el medicamento.

c) Empaque secundario: descripción y dimensiones del material que contiene, protege e identifica al producto y sus accesorios.

La definición de los elementos que conforman el producto incluye la caracterización de sus respectivos proveedores calificados. Una vez caracterizado el producto, se procedió a definir el tamaño del lote, incorporándose la información relativa a la proyección de producción en función de la demanda:

1. Cantidad de unidades del producto que conforman el lote.

2. Distribución del lote en unidades de embalaje.

3. Materiales, dimensiones y capacidad de cada unidad de embalaje.

Estas especificaciones se definieron en la Fórmula Maestra, conjuntamente con los detalles del proceso productivo a nivel industrial y las condiciones de manejo durante la elaboración del producto y su distribución. El diseño del proceso productivo se desarrolló tomándose en cuenta la secuenciación de todas las actividades relacionadas y los equipos necesarios para ejecutarlas. Se establecieron los requisitos técnicos de usuario (URS, por sus siglas en inglés) con los que se elaboró el diseño de la infraestructura de planta farmacéutica para la producción del medicamento.

\section{Sistema de Aseguramiento de la Calidad}

El sustento documental y tecnológico del Sistema de Aseguramiento de la Calidad (SAC) para la planta farmacéutica comenzó a elaborarse conjuntamente con el diseño. Su objetivo es garantizar la calidad del producto mediante el control constante de todos los procesos productivos y de apoyo, en los siguientes aspectos: Garantía de la Calidad; Prácticas Adecuadas de Fabricación (PAF); Control de la Calidad; Saneamiento e Higiene; Validación; Manejo de Quejas; Retiro de Producto; Producción y Análisis por Contrato; Autoinspección y Auditoría de Calidad; Personal; Instalaciones; Equipos; Materiales, y Documentación (OMS, 1992).

\section{Diseño de la infraestructura.}


La planta farmacéutica ha sido diseñada de manera que su infraestructura cumpla con las normas de BPM, para su calificación por parte de la autoridad sanitaria competente. La empresa proveedora de los servicios de diseño y desarrollo de plantas farmacéuticas fue seleccionada a raíz de la convocatoria general. Se verificaron, la experiencia técnica en cuanto a instalaciones farmacéuticas con las respectivas referencias de sus clientes, su estructura organizacional, sus certificaciones de entes nacionales y sus ofertas técnicas y económicas. El diseño de la infraestructura farmacéutica se basó en las URS, documento que recoge la caracterización del proceso productivo y, en consecuencia, establece: los criterios para el dimensionamiento, la definición de la tecnología y la calificación de las áreas productivas y de apoyo de la planta. Los tamaños de lote dieron una idea a los diseñadores sobre las dimensiones de los espacios, en las áreas productivas, de almacenes, áreas de tránsito de materiales, entre otros detalles técnicos.

Se estableció una proyección de existencias de materiales en almacén a tres (3) meses, con el manejo de materiales bajo el sistema "lo primero que expira es lo primero que sale" (FEFO, por sus siglas en inglés), para las materias primas químicas, y "lo primero que entra es lo primero que sale" (FIFO, por sus siglas en inglés), para los materiales de empaque primario y secundario. Las áreas de almacenamiento se diseñaron con la capacidad suficiente para el almacenamiento ordenado de materiales y productos de diversas categorías, es decir, materiales de partida y de envasado, materiales intermedios y a granel; productos acabados, en cuarentena y autorizados para la expedición, devueltos o retirados del mercado.

La información de las características del producto a ser elaborado complementó el diseño de las áreas, con respecto a sus condiciones de manejo como material en proceso y como producto terminado. De este análisis, resultó la especificación de las condiciones ambientales de las áreas que debían reinar en las áreas, así como la definición y distribución de los dispositivos de control y de seguridad en los espacios. El esquema de flujo del proceso productivo: fabricación del medicamento, envasado, etiquetado, estuchado y embalaje, resultó decisivo para la distribución de todas las áreas de planta, para lo que debió tomarse en cuenta la secuencia lógica de las actividades y la economía de movimientos. Debió tomarse en cuenta la disposición de los servicios, o sistemas de apoyo crítico, donde corresponda en cada una de las áreas de planta, para el desarrollo de las actividades respectivas. La protección al producto en proceso, al personal que operará en planta y al medio ambiente circundante, según se especifica en las normas de BPM. Al respecto, todos los sistemas de apoyo crítico se diseñaron según sus atributos de calidad acordes con los requisitos para la producción.

La distribución de equipos, accesorios, utensilios y mobiliario en las áreas se definió de tal forma que se garantizará la ejecución de todas las actividades productivas, incluyendo limpieza, de forma rápida y efectiva, evitando la contaminación cruzada. Se seleccionaron materiales que aseguraban el mantenimiento de las condiciones de calidad de las áreas y del material en proceso: con superficies lisas, de fácil limpieza, que eviten la proliferación de cargas microbianas sobre las superficies: paredes, puertas, ventanas, visores, esclusas, pisos, escaleras, luminarias, mobiliario, entre otros elementos. Las áreas de apoyo de servicios, como mantenimiento e informática, se distribuyeron en la planta de forma tal que se facilitan la logística interna para el desarrollo del proceso productivo, estableciéndose suficientemente cerca de las actividades productivas sin interferir en ellas. La proyección estimada de crecimiento de la 
planta, en cuanto al volumen de producción y a los productos que puedan desarrollarse durante su vida útil, para lo cual se tomó en cuenta una expansión de un treinta por ciento $(30 \%)$ en treinta (30) años de operación.

Con base en las premisas anteriores y en el análisis de las URS, se procedió a la elaboración del anteproyecto, que incorporó la formulación y distribución de las actividades asociadas al proyecto de diseño, construcción y adecuación de la planta farmacéutica, en las siguientes fases: Conformación del Comité Técnico que se encargó de evaluar el proyecto, por lo general, por los mismos evaluadores del Anteproyecto con las Especificaciones de Usuario. Definición de la locación donde se construirá la planta, mediante un estudio de factibilidad técnica, económica y jurídica. Formulación y presentación de la planificación del Proyecto, incluyendo el Cronograma de Actividades, con las de actividades para el desarrollo del proyecto mismo y el manejo de los recursos materiales, humanos y económicos. El proyecto contempló las especialidades: Arquitectura, Estructura, Distribuciones en Planta y Flujos de Materiales y de Personal, Instalaciones Sanitarias, Instalaciones Eléctricas, Sistemas de Aguas para Uso Farmacéutico, Sistemas de Ventilación y Acondicionamiento de Aire, Gases, Sistemas de Prevención y Extinción de Incendios y Sistemas de Video, Voz y Datos.

\section{Resultados y discusión}

\section{Información previa}

En el campo del surfactante pulmonar, el desarrollo del nuevo fármaco se inició en 1959 cuando Avery y Mead reportaron que ellos no encontraban surfactante en los extractos de los pulmones de los niños que habían muerto por la enfermedad de la membrana de hialina o síndrome de distrés respiratorio (SDR) neonatal. En base a lo cual, concluyeron que la condición de neonato prematuro y la escasez de surfactante eran los responsables de la enfermedad. Los surfactantes exógenos fueron desarrollados para prevenir y tratar el SDR en neonatos prematuros. Este tratamiento se conoce como terapia de reemplazo de surfactante (TRS) y los primeros ensayos fueron realizados hace más de 15 años. Desde 1990, fecha en que la Administración de Alimentos y Drogas (FDA, por sus siglas en inglés) aprueba el uso de varias preparaciones de surfactantes naturales y semi sintéticos, la TRS ha demostrado eficacia clínica. Varias publicaciones muestran la superioridad de los surfactantes pulmonares naturales sobre los semisintéticos.

Los surfactantes exógenos naturales actualmente disponibles en el mercado, incluyen compuestos derivados de fuentes animales, de porcinos o bovinos. La forma en que se presentan estos productos es, generalmente, liofilizada o líquida para administración intratraqueal. Estas preparaciones pueden ser de dos tipos: Surfactantes Pulmonares modificados y no modificados, cuyos precios en el mercado oscilan entre 400 y 900 dólares por dosis.

\section{Estimación de la demanda y evaluación del arte previo.}

El análisis de los registros correspondientes a los países de la región (Figura 1), arroja una demanda potencial estimada del surfactante pulmonar en aproximadamente 450.000 viales por año de $120 \mathrm{mg}$., y de 300.000 viales por año de $240 \mathrm{mg}$. Se evaluaron las otras presentaciones 
comerciales disponibles en el mercado, estudiando la forma de presentación y las dosis estimadas por kilogramo de peso del neonato. Con base en los requerimientos del surfactante pulmonar en los países del ALBA (45.000 dosis / año), se definió un tamaño estándar de 300 dosis de 120 mg. por lote. Este dato fue determinante en el diseño del proceso productivo y de la factibilidad de su desarrollo.

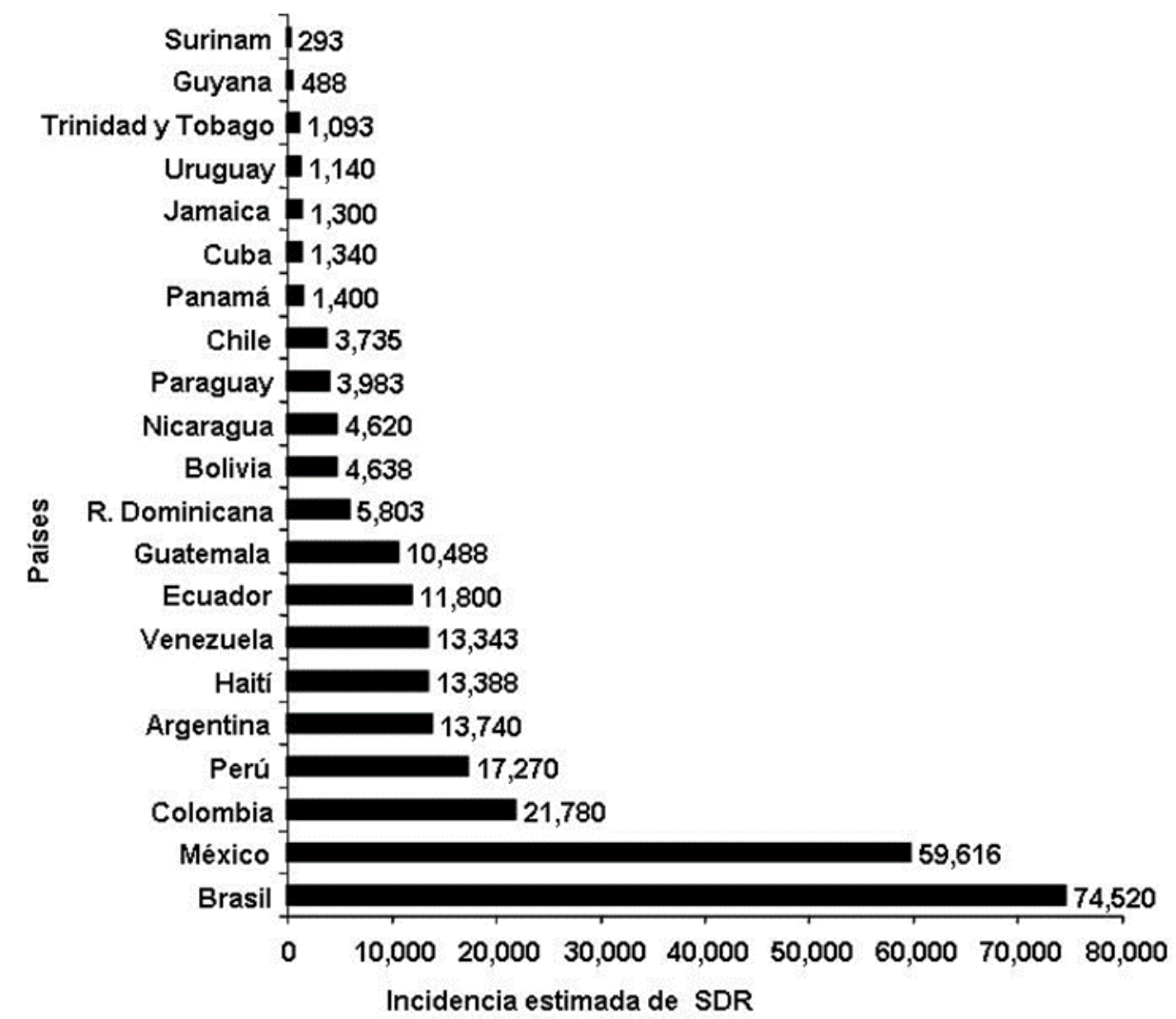

Figura 1. Estimado de la incidencia del SDR en algunos países de América Latina y el Caribe

\section{Investigación y desarrollo. Transferencia de tecnología.}

\section{Diseño del producto y diseño del proceso productivo}

El medicamento desarrollado consiste en una sustancia biológica liofilizada, clasificada como polvo estéril para reconstituir (INVIMA, 2002), extraído del lavado broncoalveolar de porcinos (lavado pulmonar), formulado para resuspender en el momento de su aplicación en $3 \mathrm{ml}$. de agua para inyectables, hasta obtener una suspensión, para ser suministrada por vía endotraqueal. El producto diseñado consta de un (1) vial de $10 \mathrm{ml}$. de vidrio color cristal con 120 mg. de medicamento, con tapón de goma y sello de aluminio, acompañado por una (1) ampolla de $3 \mathrm{ml}$. con agua estéril para inyectables, sus accesorios: una jeringa de $5 \mathrm{ml}$. y una sonda endotraqueal para neonatos, y su respectivo prospecto con las instrucciones para su uso, todo ello en un empaque, conformado por un nido de PVC para alojar a cada componente y un estuche identificado con el nombre y composición del producto, número de lote, fecha de elaboración y fecha de vencimiento. 
El empaque se diseñó para ser suficientemente resistente a los impactos durante su manipulación. El análisis conducente a la definición del lote estándar se efectuó con base en el objetivo de producción anual, de 45.000 unidades de Surfactante Pulmonar. Esto implica una producción semanal de 1125 dosis; para el proceso productivo, se estimó un requerimiento de 1013 pares de pulmones, estableciéndose un estándar de producción de 280-300 dosis por lote, resultando en un requerimiento de entre 252 y 300 pares de pulmones. Estos datos se han estimado con base en la eficiencia esperada del proceso industrial, en función de las mermas que se generen durante el proceso mismo.

\section{Obtención de la materia prima biológica.}

Para el proceso productivo, se definió cada unidad de materia prima biológica como un par de pulmones integrado a su tráquea. Este material tiene una vida útil de 4 horas, convirtiéndolo en un elemento crítico del proceso. Cada par de pulmones debe provenir de cerdos certificados en granja y matadero, sin lesiones en superficies externas y con un diagnóstico clínico, serológico y molecular negativo para los agentes infecciosos causantes de las enfermedades respiratorias de los porcinos.

Los proveedores de esta materia prima son mataderos industriales, certificados por la autoridad sanitaria (MPPS), en cuanto a Buenas Prácticas de Manufactura de alimentos, así como por la autoridad ambiental (MINAMB). Durante el desarrollo de este proyecto, se definieron dos (2) proveedores calificados, de los cuales, se seleccionó el más cercano a la planta, sin menospreciar al otro, que podría ser de utilidad posteriormente. Para el matadero seleccionado, se desarrolló un estudio de Análisis de Riesgos y Puntos Críticos de Control (HACCP, por sus siglas en inglés), para su implementación en toda el área de planta; siguiendo lo establecido en la Guía para la Inspección de Fabricantes para Productos Biológicos (OMS, 1997); se verificaron aspectos claves del SAC de la empresa, tales como: idoneidad de las áreas de planta, existencia de certificados de salud y programas de vacunación actualizados, aplicación de medicamentos y desparasitación de los cerdos llevados a beneficio, programas sanitarios en zonas críticas de la planta, control de calidad de aguas y efluentes, entre otros, y se eligió, de entre los proveedores de ganado porcino para el matadero, una granja igualmente certificada por las autoridades competentes, verificándose en ella los siguientes parámetros críticos (OMS, Ob. Cit.):

a) Localización: La granja está alejada de cualquier centro urbanizado y tiene el espacio suficiente para la cantidad de animales que alberga la explotación.

b) Distribución en Planta: La finca es una explotación de tres sitios con el sistema "todos dentro/todos fuera", provista de corrales con superficies dimensionadas.

c) Bioseguridad: La granja cuenta con procedimientos aprobados y con personal calificado para el manejo seguro de los animales, mantenimiento de las áreas, disposición de cadáveres y desechos.

d) Sanidad: La granja posee y aplica programas para la atención veterinaria de los animales criados: vacunación, desparasitación, aplicación de antibióticos y otros medicamentos, con base en procedimientos aprobados.

\section{Procedimiento de obtención de surfactante pulmonar.}


Se establecieron los parámetros de composición del surfactante en base a los trabajos pioneros en surfactante, en los cuales se reporta cuáles son los porcentajes de los fosfolípidos y proteínas de la mezcla. Se diseñó un procedimiento basado en filtraciones y cromatografias del lavado bronco alveolar de porcinos, que permitieron llegar a la composición esperada para el surfactante pulmonar. La composición de fosfolípidos se evaluó cuantitativamente mediante la determinación de fósforo y se estableció un rendimiento de $120 \mathrm{mg}$ por par de pulmones en promedio y el análisis cualitativo se llevó a cabo por cromatografía de capa fina encontrándose como componente mayoritario la Dipalmitoilfosfatidilcolina. La determinación de la concentración de proteínas se realizó por el método colorimétrico (BCA) obteniéndose como resultado $1,7 \mathrm{mg} / \mathrm{ml}$. La identificación de estas proteínas se realizó por electroforesis en geles de poliacrilamida y transferencias seguidas de inmunotinción con los anticuerpos específicos para las proteínas SP-B y SP-C, la cuales fueron identificadas.

Los datos teóricos provenientes de las proyecciones de producción sirvieron de base para la definición de los parámetros asociados al proceso de escalamiento industrial. Los datos de capacidad de producción planteados como objetivo de la planta, se emplearon para evaluar el rendimiento y eficiencia del proceso de producción. De esta forma se estableció el rendimiento del proceso por cada par de pulmones, con base en la tecnología de extracción en prototipos para pruebas a nivel de laboratorio. Se evaluó cada paso en el proceso de obtención de surfactante empleando modelos de equipos de laboratorio, escalables a equipos industriales ya existentes en el mercado, con lo cual se ha obtenido un desarrollo en continuo del proceso. Esto permitió establecer el requerimiento de cada insumo por lote de surfactante pulmonar.

\section{Sistema de Aseguramiento de la Calidad}

El Sistema de Aseguramiento de la Calidad se está desarrollando actualmente, según el cronograma que establece la elaboración de procedimientos, instructivos de trabajo, formatos, protocolos de validación y otros documentos que apliquen para todos y cada uno de los ámbitos de operación de la planta, desde la calificación de los proveedores hasta el control de la cadena de distribución. La autoridad sanitaria competente, el Ministerio del Poder Popular para la Salud (MPPS), realizará inspecciones programadas, verificando el avance del SAC de la planta y el estado de calificación de la planta, con miras al otorgamiento y posterior renovación de la certificación de Buenas Prácticas de Manufactura, indispensable para su funcionamiento.

\section{Diseño de la infraestructura de planta}

El diseño y desarrollo de la planta lo realizó la compañía LC \& LP Arquitectura e Ingeniería, C. A., empresa nacional dedicada a proyectos de infraestructura, con una nutrida experiencia en instalaciones farmacéuticas, reconocida a nivel nacional. Esta empresa desarrolló el diseño de la infraestructura de planta, con base en las especificaciones de requerimientos de usuario, documento creado a raíz de los resultados obtenidos en la fase de escalamiento industrial, que recoge la caracterización del proceso productivo y, en consecuencia, indica los criterios para el dimensionamiento, la definición de la tecnología y la calificación de las áreas productivas y de apoyo de la planta. La planta fue diseñada en cumplimiento con lo establecido en las normativas farmacéuticas e industriales vigentes, entre las que se encuentran, Normas de 
Buenas Prácticas de Manufactura, específicamente en lo referente a las instalaciones, Norma ISO-14644, relativa al diseño de áreas farmacéuticas con ambientes controlados, Normas relacionadas con las condiciones de calidad de servicios, Normas de higiene y seguridad industrial, tales como las de iluminación, ruidos, ergonomía, dispositivos y sistemas de seguridad y almacenamiento y transporte de materiales peligrosos.

En resumen, la planta diseñada cuenta con una sección productiva y una sección de apoyo, con áreas físicamente separadas entre sí, para garantizar la protección al producto en proceso, al personal y al medio ambiente externo a la planta. La sección productiva se diseñó para que el proceso productivo se efectúe en forma secuencial, de forma similar a la mostrada en la Figura 2 y según se especifica en la Fórmula Maestra, en áreas clasificadas según su función, con base en la concentración máxima de partículas en el aire (en partículas por pie cúbico), delimitadas físicamente y distribuidas de tal forma que cada actividad precedente esté suficientemente cerca de la subsiguiente, sin que haya cruzamiento de materiales que pueda contaminar el producto.

\section{Esta sección está compuesta por las siguientes áreas:}

a) Andén de recepción de materiales, abierto al exterior de la planta.

b) Esclusa de materiales, para su ingreso a planta: Con clasificación 100.000 y flujo de aire desde la planta hacia el exterior, separada por compuertas a ambos extremos, para que el personal de recepción ingrese el material desde el andén, sin ingresar a planta, y el personal respectivo de planta, según la naturaleza del material (materia prima biológica, materia prima química y materiales de empaque) lo retire de la esclusa para su traslado a las áreas correspondientes.

c) Zona de vestuarios y sanitarios para el personal interno de planta, en la cual ese personal efectúa su muda de ropa a la indumentaria reglamentaria, y su limpieza y sanitización previas a su ingreso a la zona productiva (Figura 3). Esta zona tiene clasificación 100.000 y flujos de aire entre las secciones, desde la zona productiva hacia el exterior y los extractores de aire en las salas sanitarias.

d) Almacenes de materiales: En estos espacios, físicamente delimitados y separados entre sí, se almacenarán las materias primas químicas y los materiales de empaque. Están diseñados para el mantenimiento de existencias para los procesos productivos en planta, por tres meses. Poseen clasificación ambiental 100.000, con flujo de aire positivo hacia los pasillos de planta.

e) Pasillos internos, para el desplazamiento de materiales y de personal en la sección productiva de la planta, con clasificación ambiental 100.000.

f) Áreas productivas, donde se desarrollan las actividades de producción: pesada, fabricación y llenado de medicamentos, así como las de control de calidad: laboratorio físico-químico y laboratorio microbiológico. Estas áreas poseen clasificaciones ambientales más rigurosas, que van desde la 10.000 hasta la 100 (áreas estériles), separadas físicamente de las áreas adyacentes por esclusas de materiales y de personal, con flujo de aire positivo hacia el exterior de las mismas, a través de las esclusas.

g) Áreas de empaque, consideradas también como áreas productivas, aunque en las mismas se maneja una clasificación ambiental 100.000. En ellas, se efectúan las actividades de 
inspección óptica de ampollas, etiquetado de envases (viales y ampollas), codificación de empaques, estuchado y embalaje.

h) Almacenes de productos terminados, con clasificación ambiental 100.000, con capacidad para el mantenimiento de lotes por tres meses.

i) Esclusa de productos terminados, para su traslado al andén de carga, separado de la esclusa de materiales, para evitar el cruzamiento con el material que ingresa a planta.

j) Andén de carga, para la salida de los lotes de productos terminados a la cadena logística, físicamente delimitado del andén de recepción de materiales.

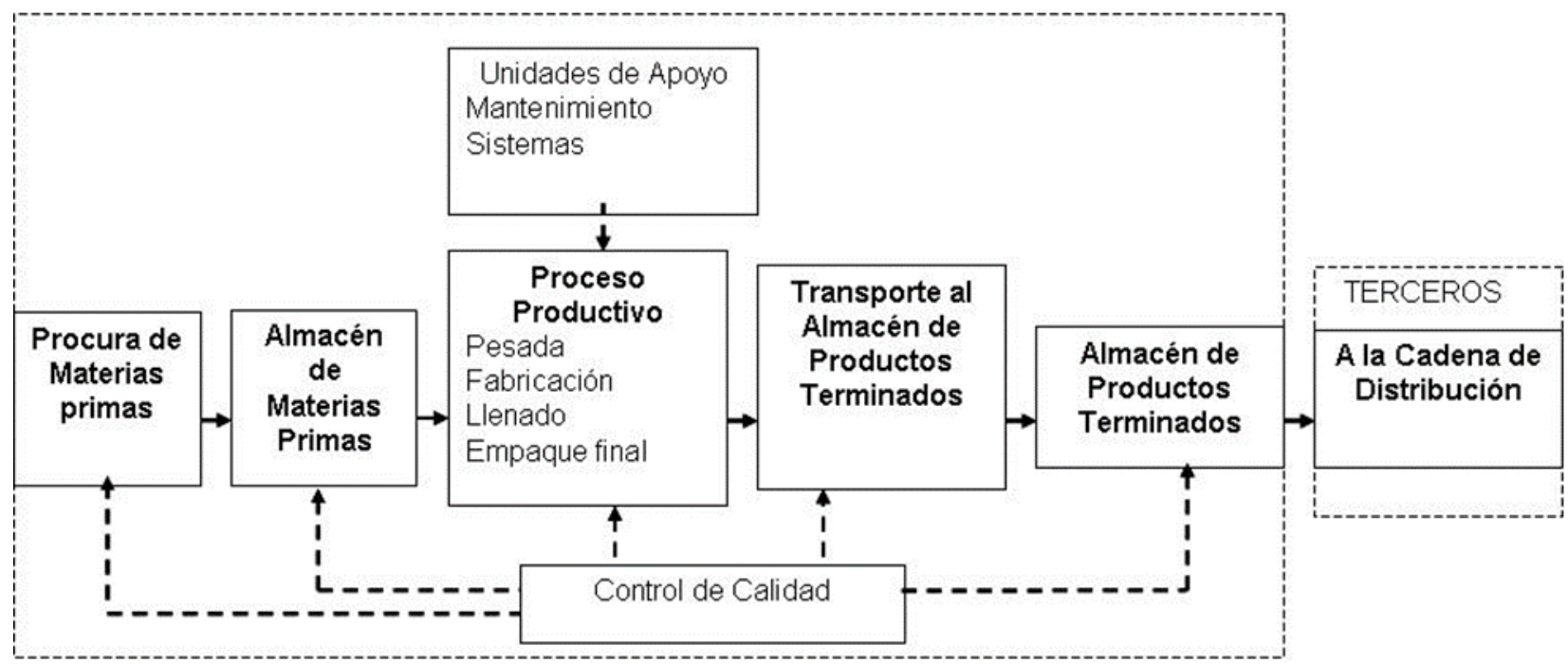

Figura 2. Diagrama de flujo típico del proceso productivo de medicamentos.

La sección de apoyo se diseñó bajo la premisa de que las áreas que la componen fueran lo menos invasivas posible hacia los procesos productivos, por lo que las mismas han sido ubicadas suficientemente cerca de la sección productiva, aunque físicamente separadas entre sí. Entre las áreas de esta sección, se encuentran:

a. Áreas de servicios de equipos, donde se ubican las partes traseras de equipos como liofilizadores.

b. Áreas para el mantenimiento de luminarias, tuberías de servicios, ductos de aire acondicionado y bancos de filtros absolutos de aire. Estas áreas de mantenimiento se emplazaron sobre el techo de las áreas productivas respectivas (Figura 4).

c. Áreas para servicios generales, tales como lavamopas y lavandería, adyacentes a los pasillos de planta, así como las áreas de almacenamiento de desperdicios, debidamente identificadas según la naturaleza y peligrosidad de los materiales de desecho que se generen, ubicadas en las afueras del edificio de planta.

d. Salas de máquinas, fuentes de los diferentes servicios de apoyo crítico de la planta: aire acondicionado, agua tratada, agua con calidades farmacéuticas y vapor con calidades farmacéuticas, establecidas dentro del edificio de planta, y energía eléctrica, vapor saturado, aire comprimido, vacío y gases, ubicadas en las afueras de la edificación, por razones de seguridad.

e. Salas de control de los sistemas automatizados y dispositivos de seguridad. 
f. Planta de tratamiento de aguas residuales, diseñada para el procesamiento del agua servida proveniente de las actividades productivas y por el uso sanitario.

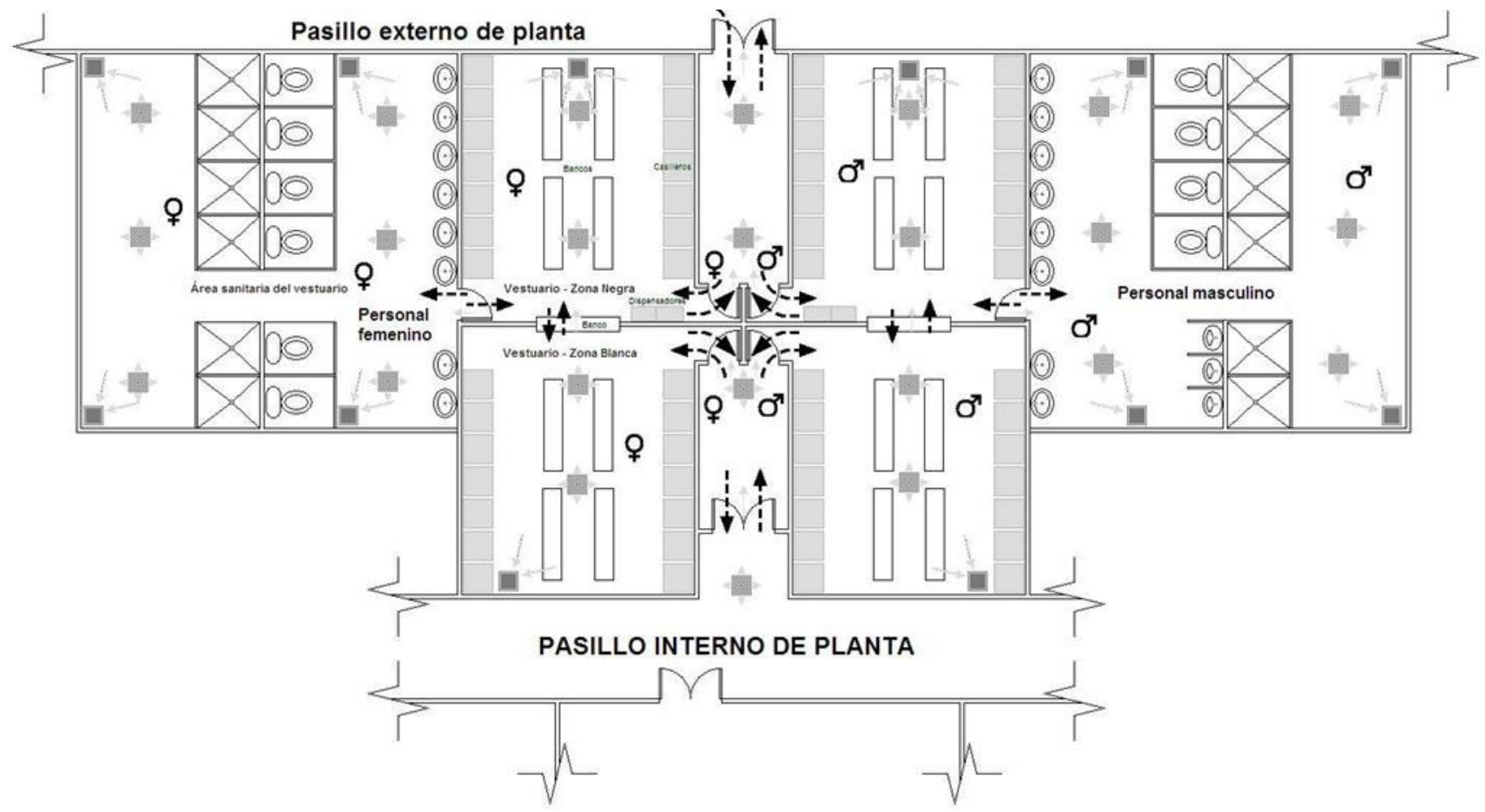

LEYENDA

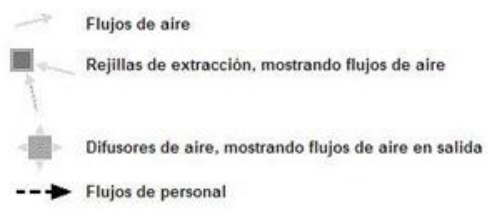

Figura 3. Esquema básico de distribución de flujos de aire y de personal en áreas de vestuarios, antesala a zonas productivas farmacéuticas.

Tratándose de un producto de origen biológico todos los proveedores de materiales deben estar calificados, según las pautas establecidas en el SAC de la planta. En cuanto a los proveedores del material biológico, los pulmones de porcinos, se considera la distancia entre la planta y cada proveedor como un factor de localización vital, por la vida útil de la materia prima. Tomándose en cuenta que el proceso productivo no debería depender de un solo proveedor, se optó por establecer, en las cercanías del matadero, una instalación industrial que opere como estación para el proceso de extracción del surfactante de los pulmones, y enviar el material en un tanque refrigerado a la planta. 


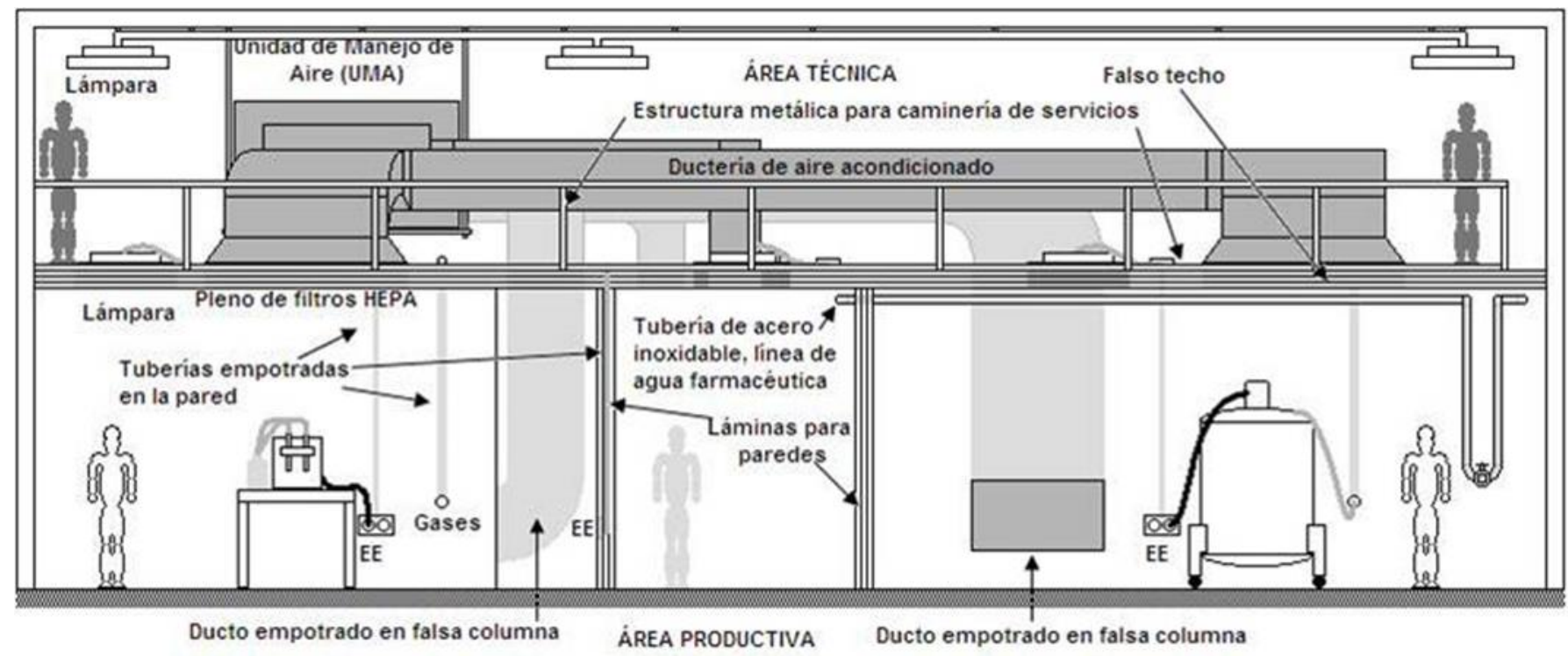

Figura 4. Esquema básico de distribuciones de áreas técnicas y productivas y de algunos servicios. EE: Energía Eléctrica. Gases: El (los) gas(es) que aplique(n) en cada área: aire comprimido, nitrógeno, gas natural, $\mathrm{CO2}$, vacío.

El siguiente elemento en la cadena logística es el medio y el procedimiento para el transporte del lote de pulmones a la estación de extracción de surfactante. Se definieron las dimensiones y los componentes del contenedor tipo para pulmones y el número requerido de contenedores para el traslado seguro del lote, así como las características técnicas del vehículo, que debe ser apto para el traslado del material manteniendo la cadena de frío. Se realizó un diseño de la estación de extracción de surfactante como un módulo tipo móvil y flexible, de fácil instalación, acondicionamiento a los requerimientos de BPM y remoción, para que la misma pueda ser emplazada cerca de cualquier proveedor calificado, aprovechando los recursos de esa empresa o de la misma zona industrial para sus operaciones, pudiendo incluso disponer de fuentes propias de energía.

La instalación deberá estar compuesta por las siguientes áreas: Muelles de carga y descarga de materiales. Esclusas de materiales, definidos y físicamente separados, para la materia prima biológica (contenedores de pulmones), y para los tanques refrigerados para el traslado del producto en proceso a planta y recipientes con las sales pesadas en la planta, todas con clase 100.000. Vestuarios para el ingreso del personal al interior de la estación, concebidos de forma similar a los de la planta, con clase 100.000. El área de procesamiento de pulmones, clase 10.000, provista del sistema para lavado pulmonar, y el suficiente mobiliario para la estadía de dos operarios y los contenedores de pulmones. Las temperaturas en esta área deben estar controladas, de tal forma que, en torno a las máquinas de extracción de surfactante, la temperatura sea de cuatro grados centígrados $\left(4^{\circ} \mathrm{C}\right)$, dentro de campanas de flujo laminar con cortinas de vinilo, y para protección al personal, una temperatura de dieciocho grados $\left(18^{\circ} \mathrm{C}\right)$. El área de elaboración de solución salina, clase 10.000, con temperatura controlada, donde se emplazará el sistema de generación de agua purificada y dos tanques reactores de acero inoxidable 316L, con camisa del mismo material y control de temperatura, con el suficiente espacio y mobiliario para un operario, que incluya también el almacén temporal de los contenedores de la materia prima química para la elaboración de la solución. El área técnica, 
donde se establecerán los sistemas de apoyo crítico (aire acondicionado, agua purificada y agua helada) y los centros de control automatizado del sistema de lavado pulmonar, adyacente y físicamente separada de las áreas productivas de la estación.

El siguiente punto es el transporte de la solución de surfactante obtenida en la estación. Se seleccionó un tanque contenedor comercial, automatizado con control de temperatura y agitación para ser transportado en un vehículo de carga tipo cava, con control de temperatura para mantener en su interior el lavado pulmonar a cuatro grados centígrados $\left(4^{\circ} \mathrm{C}\right)$, contenida en bolsas colapsables estériles, y mangueras y conectores sanitarios de alta tecnología, para acoplar el tanque con la red de tuberías de acero inoxidable 316L del área de filtrado, a través de un receptáculo sanitario empotrado en pared.

El proceso para la creación y puesta en marcha de un laboratorio farmacéutico conlleva una inversión muy importante en tiempo y recursos, con miras a la obtención de una infraestructura y un Sistema de Aseguramiento de la Calidad con plena capacidad para cumplir con los lineamientos establecidos en la normativa competente y, en consecuencia, para garantizar la calidad de los productos que en ella se elaborarán, la salud y la seguridad de todo el personal que en ella laborará, y la minimización de los impactos que sus actividades causen al medioambiente y a la comunidad, en el lugar en donde se establezca. Desde la concepción de la idea, hasta la certificación de la infraestructura a cargo de la autoridad sanitaria competente, todo el proyecto debe ser documentado. Además, el SAC de la empresa en torno a la cual operará la planta debe sustentarse en toda esta documentación generada.

\section{Agradecimientos}

Los autores quieren agradecer al FONACIT por el financiamiento del presente trabajo a través de los Fondos del proyecto Misión Ciencia No 2000001361, titulado "Desarrollo de la Tecnología para la producción de surfactante Pulmonar".

\section{Bibliografía}

Asamblea Nacional [Ley sobre sustancias, materiales y desechos peligrosos]. Gaceta Oficial de la República Bolivariana de Venezuela No. 5.554 Extraordinaria. (2001 Noviembre 13)

Been J.V., y Zimmermann L. (2007). What's new in Surfactant? A clinical view on recent developments in neonatology and paedriatics. Eur J Pediatr.; 166: 889-899. DOI 10.1007s00431-007-0501-4.

Clements, J. A., y Avery, M. E. (1998). Lung Surfactant and Neonatal Respiratory Distress Syndrome. Nueva York: American Journal of Respiratory and Critical Care Medicine. Vol. 157: S59-S66.

Comisión Venezolana de Normas Industriales (COVENIN) (2002). Norma COVENIN-ISO TR 10013:2002. Caracas. 
Cullen, P. J.; Guzmán, B.; Matías, D. R., y Martínez, M. A. (2007). Surfactante Pulmonar. Vacunación Hoy. Vol.15 (85): 19-28. Ciudad De México.

Ghodrat M. (2006) Lung Surfactants Formulary Review. AM J Health-Syst Pharm. Vol. 63:15041521. Atlanta.

Instituto Nacional de Higiene Rafael Rangel (INHRR) (2008). N-PERC-001: Norma para el Registro, Liberación de Lotes y Control de los Productos Biológicos. Caracas.

Instituto Nacional de Vigilancia de Medicamentos y Alimentos (INVIMA) (2002). Manual de Normas Técnicas de Calidad: Guía Técnica de Análisis. Bogotá: Ministerio de Salud, República de Colombia.

International Conference on Harmonization of Technical Requirements for Registration of Pharmaceuticals for Human Use (ICH) (2008). Pharmaceutical Quality System (Norma Q10). Ginebra, Suiza.

International Standardization Organization (ISO). Cleanrooms and Associated Controlled Environments - Part 4: Design, Construction and Start-Up (Norma ISO 14644-4). Ginebra, Suiza.

Ministerio de Salud y Desarrollo Social [Resolución mediante la cual se dictan las Normas de Buenas Prácticas de Distribución de Medicamentos]. Gaceta Oficial de la República Bolivariana de Venezuela No. 37.966. (2004 Junio 23)

Ministerio de Salud y Desarrollo Social [Resolución por la cual se adopta el Manual de Buenas Prácticas de Manufactura para la Fabricación de Productos Farmacéuticos (BPM) de la Organización Mundial de la Salud (OMS)]. Gaceta Oficial de la República Bolivariana de Venezuela No. 38.009. (2004 Agosto 26)

Ministerio del Ambiente y de los Recursos Naturales [Decreto 2.635 referente a las "Normas para el Control de la Recuperación de Materiales Peligrosos y el Manejo de los Desechos Peligrosos"]. Gaceta Oficial No. 5.245 Extraordinaria. (1998 Agosto 03). Extraído el 29 de junio de 2008 desde

Oficina Española de Patentes y Marcas. Método para solubilizar una proteína surfactante pulmonar. $\mathrm{N}^{\circ} 2176503 \mathrm{~T} 3$.

Organización Mundial de la Salud (OMS) (1997). Guía para la Inspección de Fabricantes de Productos Biológicos. Ginebra: Autor.

Presidencia de la República [Decreto 638 del 26 de abril de 1995, sobre las "Normas sobre Calidad del Aire y Control de la Contaminación Atmosférica"]. Gaceta Oficial No. 4.899 Extraordinario. (1995 Mayo 19). 
Presidencia de la República [Decreto 883 del 11 de octubre de 1995, sobre las "Normas para la Clasificación y el Control de la Calidad de los Cuerpos de Agua y Vertidos o Efluentes Líquidos”]. Gaceta Oficial No. 5.021 Extraordinario. (1995 diciembre 18).

Ramírez Carrasco, Francisco (2003). Valoración de la Congruencia Espacial entre la Actividad Residencial y Terciaria en el Centro Urbano de Barcelona. Tesis doctoral. Universidad Politécnica de Cataluña. España.

Rangel Infante, Vicente Emilio (2009). Modelo de Logística Inversa para la Recolección de Lotes de Productos Farmacéuticos No Conformes: Clasificación, Tratamiento y Disposición Final de Sus Componentes. Caso: Laboratorios Verifar, C. A. Tesis de Maestría. Tesis de Maestría no publicado. Universidad Nacional Experimental Politécnica "Antonio José de Sucre". Venezuela.

Sánchez, C., y Torres, J. (2004) Surfactante Pulmonar. Santiago de Chile. Rev. Ped. Elec [en linea]. Vol 1. N¹. ISSN 0718-0918: 45-50.

Taeush HW, Karen LU, Ramirez-Schrempp D. (2002). Improving Pulmonary Surfactants. Acta Phamacol Sin; 23 supplement: 11-15. 